\title{
Menstrual Dysfunction and Treatment among Adolescents with Congenital Heart Disease
}

Maayan Leroy-Melamed, MD ${ }^{1}$ Amy Katz, MPH ${ }^{1}$; Marcia L. Shew, MD, MPH ${ }^{1}$

${ }^{1}$ Indiana University School of Medicine

Funding: Dr. Leroy-Melamed is supported by a postdoctoral training grant (T32) of the National Institutes of Health under award number 2T32GM008425-26.

Acknowledgements: A poster presentation of this work was given at the Annual Meeting for the Society for Adolescent Health and Medicine in Seattle, WA on March 7, 2019.

\section{Contact:}

Maayan Leroy-Melamed, MD

410 W. $10^{\text {th }}$ St, Suite 1001

Indianapolis, IN 46205

mmelamed@iu.edu

Office: 317-274-8812

Fax: 317-274-0133

\section{Word Count:}

Abstract: 239

Main text: 2358

\section{Abbreviations:}

AYA $=$ Adolescents and Young Adults

$\mathrm{CHD}=$ Congenital Heart Disease

COC $=$ Combined Oral Contraceptive pills

DMPA = Depot Medroxyprogesterone Acetate

$\mathrm{EMR}=$ Electronic Medical Record

NSAID = Non-Steroidal Anti-Inflammatory Drug

OCPs $=$ Oral Contraceptive Pills

OTC = Over the Counter

$\mathrm{SRH}=\mathrm{Sexual}$ and reproductive health

This is the author's manuscript of the article published in final edited form as:

Leroy-Melamed, M., Katz, A., \& Shew, M. L. (2020). Menstrual Dysfunction and Treatment Among Adolescents With Congenital Heart Disease. Journal of Pediatric and Adolescent Gynecology, 0(0). https://doi.org/10.1016/j.jpag.2020.08.012 


\section{Abstract}

Study Objective: This study describes menstrual dysfunction and treatment among adolescent and young adult (AYA) females with congenital heart disease (CHD). Design: Data collected from a one-time survey completed by AYA females (and mothers if AYA unable).

Setting: Participants were recruited from pediatric cardiology clinics.

Participants: Female AYA with CHD, ages 14-21 ( $\mathrm{N}=114)$.

Interventions: None.

Main Outcome Measures: The questionnaire assessed sexual and reproductive health $(\mathrm{SRH})$ concerns, behaviors and management. Outcome measures were self-reported menstrual complaints, use of over the counter (OTC) pain relief medicines for dysmenorrhea, reported visits with a clinician for a menstrual problem and reported use of hormones for menstrual problems or birth control.

Results: Mean age was 17.0 years $(S D=2.2)$. A majority $(83 \%)$ reported one or more menstrual complaints: $67.5 \%$ cramping, $42.1 \%$ irregular menses, $46.5 \%$ heavy periods, and $88 \%$ reported any history of taking OTC for pain relief. Increased menstrual complaints were not associated with level of cardiac complexity, reported transplant, or reported use of hormonal contraception. However, $32 \%$ of participants reported use of hormonal contraception for menstrual dysfunction; combined oral contraceptive pills (COCs) were the most common; two of these women carried contraindications to estrogen. 
Conclusions: A large majority of AYA females with CHD reported menstrual dysfunction. Use of OTC medication for menstrual pain and inappropriate use of estrogen creates concerns that menstrual disorders may be unaddressed or addressed inappropriately. Thus, gynecological needs of adolescents with CHD may need to be specifically targeted by providers that feel comfortable with this population and their complex needs.

Keywords: Menstruation Disturbances, Cardiovascular Abnormalities, Contraindications 


\section{Introduction:}

Over $85 \%$ of adolescents and young adults (AYA) with congenital heart disease (CHD) are living well into adulthood and are in need of reproductive health. ${ }^{1}$ AYA females with CHD have specific contraception needs due to increased risk of thromboembolic events and/or fatality, as well as complications with unintended pregnancy. ${ }^{2-4}$ Use of certain medications - both over the counter (OTC) and prescribed - for menstrual problems need to be monitored due to potential adverse events associated with the CHD condition or transplantation. Sexual activity is rarely assessed in women with CHD; data suggests that AYA with CHD have inadequate knowledge of contraception and that few have discussed sexual and reproductive health $(\mathrm{SRH})$ issues with their cardiologists or any other health professional. ${ }^{5-10}$ These findings raise the need for interventions that address these issues. ${ }^{11,12}$

The exact reproductive health needs of adolescents with CHD, including those that have been transplanted due to a CHD related lesion, are not well described despite the literature continuing to report poor provisions of services particularly in the areas of family planning, counseling about pregnancy, and treatment for menstrual dysfunction, including dysmenorrhea, menorrhagia and irregular menses. A few studies have reported increased rates of menstrual dysfunction, including delayed menarche, anovulatory cycles and dysmenorrhea in AYA females with CHD. ${ }^{5-7,9}$ Others have reported increased rates of menorrhagia as a significant complication in AYA females using anticoagulants. ${ }^{8}$ As with other chronic diseases, the reproductive health needs may be more complex and unique, requiring a better understanding not only of the CHD pathophysiology, but also prevalence of menstrual dysfunction in $\mathrm{CHD}{ }^{9}$ 
In attempts to better understand more current menstrual related needs, this study examines the self-reported menstrual dysfunction among AYA females with CHD and treatment with OTC and provider-prescribed medications, including hormonal contraception. Secondary analysis examined the associations of menstrual dysfunction with OTC and hormonal treatments, history of sexual activity, transplantation, and cardiac complexity.

\section{Materials and Methods}

\section{Study Participants.}

This study was part of a larger project that recruited adolescent females with $\mathrm{CHD}$, as well as their mother or female guardian, from two university associated pediatric cardiology clinics from September 2015 to August 2018. Eligible adolescents between the ages of 14 and 21 years old, and the mother or female guardian of eligible adolescents, were simultaneously recruited at their regularly scheduled appointments. Recruitment of only female guardians was related to survey items that examined specific daughter-mother reproductive conversations (not used in this study). Literature suggests that conversations surrounding puberty and sexuality occur more commonly amongst adolescent females and their mothers, rather than their fathers. ${ }^{13-15}$ Informed assent and/or consent were obtained from interested participants and their mother/guardian. Parents provided permission for adolescents who were 17 years and younger, and young adults aged 18 years or older provided their own informed consent. Recognizing that some potential participants would not have the ability to complete a 
survey autonomously, the guardian was asked in all cases if their daughter had the ability to participate in a web-based survey at the time of recruitment. If the guardian did not feel their daughter was capable of completing a survey, the guardian was offered the opportunity to participate alone. The researcher was present during completion of the questionnaires to ensure independence of responses. If both patient and guardian completed the survey, patient data was used for this analysis due to assumed greater accuracy with regards to sexual activity, hormone use, and SRH data. However, in the cases where adolescents did not complete a survey, the parent or guardian survey was used in order to assess menstrual disorders among the entire cohort.

Consented adolescents and/or their parent/guardian independently completed a one-time electronic questionnaire assessing various reproductive issues (Table 1). Participants answered yes/no to reports of cramping, irregular menses, and/or heavy periods. Menstrual problems were not defined, and were left to the participant's own interpretation. Participants reported if they had ever seen a clinician for a menstrual problem and were asked about use of OTC medication for pain (menstrual cramps). All of those queried were asked about use of any hormonal contraception for a menstrual problem or puberty. If a participant reported use of hormonal contraception, they were asked to indicate which method(s) they used from a list provided and if use was in the past, currently using, or never used. Only the adolescent survey asked about sexual activity (yes/no) as well as if the use of this hormonal contraception was to prevent pregnancy. Cumulative use of hormonal methods for the entire cohort was examined by combining reports of use for menstrual dysfunction and/or pregnancy prevention. 
The study cardiologist completed a clinician-generated survey for each participant by accessing the patient's Electronic Medical Record (EMR) to report the adolescent's CHD lesion(s) and current contraindications to hormonal contraceptive methods. Prior to enrollment, a predefined list of contraindications to birth control was compiled based on current literature, national and international risk criteria, and /or consensus guidelines for congenital cardiac lesions. ${ }^{4-6,16-20}$ Contraindications to estrogen included adolescent women with functional class III and IV heart failure, cyanosis, Eisenmenger syndrome, history of thromboembolism, non-repaired coarctation and pulmonary arterial hypertension. For analysis, cardiac lesions were further classified by the study cardiologist as simple, moderate, complex or transplanted based on American College of Cardiology's criteria. ${ }^{21}$ Participants and parents were asked to report their (or their daughter's) level of disability on the surveys as none, mild, mild to moderate, moderate to significant, or significant with regards to level of assistance and independence with responsibilities and activities of daily living. These were grouped into none, mild to moderate and moderate to significant for data analysis.

\section{Statistical Procedure.}

Data was analyzed using Statistical Package for Social Sciences (version 26). Descriptive statistics include population characteristics, which are reported as percentages. All adolescents that had reported (or parents who reported for their daughter) having experienced menarche were included in this analysis. Bivariate analyses (chi-squared and t-tests) were used to examine factors associated with menstrual dysfunction and treatment.

\section{Results}


A total of 95 adolescent/parent pairs and 15 individual female AYA, and 16 individual parents were enrolled to participate in this study. For this analysis, 1 participant was excluded due to age, 6 due to incomplete surveys, and 4 who had not yet achieved menarche. Fifteen parental surveys were used (one excluded due to no menarche) without an AYA survey due to disability (Figure 1). Thus, our final sample consisted of 114: 99 AYA and 15 parent surveys.

The average age of the AYA was 17.0 (range 14 to 21) years at time of enrollment and 12.5 (range 8 to 17) years at menarche. Baseline demographic information is included in Table 2. A majority reported having no disabilities $(\mathrm{N}=80$, 71\%), 22 (19.5\%) participants reported mild to moderate disability, 11 (9.7\%) parents reported moderate to significant disability, and 1 participant did not answer the question about disability. Forty-six (40.4\%) had a single cardiac lesion reported by the clinician, and $68(59.6 \%)$ had more than one cardiac lesion. Twenty-eight (24.6\%) were classified based on their current lesion as having simple CHD lesions, 46 (40.4\%) had moderate, $33(28.9 \%)$ had complex lesions, and $7(6.1 \%)$ were transplanted.

Menstrual dysfunction was prevalent (Table 1). Ninety-five participants (83.3\%) had at least one menstrual complaint, 77 (67.5\%) reported cramping, 48 (42.1\%) irregular menses, and 53 (46.5\%) heavy menses (Figure 2). Twenty-nine percent of all participants $(\mathrm{N}=33)$ reported seeing a clinician for some type of menstrual dysfunction. About a quarter of those who had menstrual problems or saw a clinician for a menstrual problem had a contraindication to estrogen (Figure 2). All participants with confirmed transplanted hearts $(n=7)$ reported at least one menstrual complaint as well. There was no association with self-reported menstrual dysfunction and seeing a clinician for that 
problem $(p=.78)$ or with level of disability $(p=.97)$. Complexity of cardiac lesion was not associated with increased reports of menstrual dysfunction $(p=.69)$. However, higher lesion complexity was associated with increased likelihood of seeing a clinician for menstrual dysfunction ( $p=.03$ ). Of those who reported heavy bleeding $(N=53)$, only 4 reported current Warfarin use. Ninety-five participants - including 4 out of 7 AYA with cardiac transplant - reported use OTC medication for menstrual related pain.

Cumulative use of hormones was assessed in the cohort. Assessing combined responses by adolescent and parents to all questions (past use, current use) and use for any purpose, a total of 38 patients (33\%) had been prescribed hormonal contraception (any method) at some point. The most frequently reported hormonal method was COC pills; 25 (22\%) were using COC pills currently or had used in the past. Ten $(7 \%)$ reported past or current use of depo-medroxyprogesterone; 2 reported use of a progestin IUD, and 10 reported implant use. Only 1 patient reported use of the patch in the past. No association was found between self-reported menstrual dysfunction and reports of use of any hormonal contraception $(p=.929)$.

With regards to current use $(\mathrm{N}=26)$ of hormonal methods, a majority $(\mathrm{N}=14)$ were currently using combined oral contraceptive pills (COCs): $3(3 \%)$ by parental reports, 2 $(2 \%)$ by self-report for both menstrual problem and birth control, $9(8 \%)$ for menstrual problem only. This current use of COCs was examined due to 19 (16.7\%) participants carrying a contraindication to estrogen. One of these participants with contraindications reported current COC use, and 1 reported use in the past.

Thirty-four of the 99 (34\%) adolescent participants self-reported a history of vaginal intercourse (again, parent surveys did not include this question). Menstrual 
dysfunction was reported by 27 of the 34 who reported sexual activity, which did not differ significantly from the entire study sample ( $79 \%$ vs. $83 \%$ respectively, $p=.51)$. Of these 34 sexually active adolescents, 23 (68\%) reported having been or currently on prescribed contraception. Two participants denied sexual activity but endorsed using any birth control method to prevent a pregnancy.

\section{Discussion}

Menstrual dysfunction occurs frequently (83.3\%) in AYA females with CHD, though relatively few reported receiving clinical treatment from a provider (28.9\%). Based on the average age of menarche, delays in puberty were probably infrequent. Despite seeing a high level of reports of one or more menstrual complaints, dysmenorrhea was the most common complaint among the cohort, but the prevalence is consistent with that reported in the general population. ${ }^{22}$ Reports of heavy menses were more prevalent than that seen in the general population, whereas irregular menses was similar to the general population. ${ }^{22-24}$ Lesion complexity was not associated with more menstrual dysfunction. However, higher lesion complexity was associated with higher likelihood of medical intervention. This may indicate that increased interaction with medical providers leads to more direct referral for treatment.

Interestingly, those who reported a cardiac transplant all experienced menstrual dysfunction and over half reported the use of OTC medication. Due to the way this was asked in the survey, we could not differentiate non-steroidal anti-inflammatory drugs (NSAIDs) from other medications (such as acetaminophen). This is potentially concerning as NSAIDs carry a relative contraindication in transplanted individuals due 
to risk of renal toxicity associated with use of transplant-specific medication. ${ }^{25}$ This may raise the need to ask specifically about menstrual cramping in transplanted patients to assure that they are not accessing OTC medications that include NSAIDs.

Use of warfarin by adolescents has been associated with menorrhagia rates twice that of the general population. ${ }^{26,27}$ Although this study had high numbers of selfreported heavy menses, no association with warfarin was found. Warfarin use was not that common and this may reflect more use of other anticoagulant therapies (i.e. direct oral anticoagulants) which was not specifically asked by the survey, nor have direct oral anticoagulants been associated with heavy menses..$^{28,29}$ However, use of anticoagulants, of any type, may have played a role in the higher prevalence of heavy bleeding reported in this population.

At least $10 \%$ of the participants who reported any of the three reported menstrual complaints had contraindications to estrogen. In addition, 2 participants who were prescribed estrogen-containing contraception had a contraindication to estrogen. Previous studies have shown that providers prescribe estrogen-containing contraception to reproductive-aged patients with contraindications. ${ }^{30,31}$ Given the potential for estrogen contraindications in AYA with CHD, and the fact that $5 \%$ (2 of 38 ) had reported use of this medication in the face of a contraindication, there is a strong need for provider education when addressing reproductive needs of this population.

Finally, though the overall numbers were small, many sexually active adolescents were not prescribed contraception, raising concerns for pregnancy complications or unintended pregnancy. At times patients may already be sexually active or contemplating initiation of sex when they present to the clinician with menstrual 
complaints. Thus, it is important that providers do query, confidentially, all reasons for hormonal contraception to ensure proper response to the health care needs of the patient. While many factors may contribute to contraceptive choice, all providers may benefit from education and training of more effective, non-estrogen containing methods of contraception, such as IUDs and implants.

A limitation of our study was that results were based on self-reported or maternal-reported survey data and would benefit from clinical clarification through detailed history. We recognize this is a convenience sample; however, attempts were made to recruit any adolescent who qualified during a clinic visit when study researcher could be present. In addition, the study was predominantly Caucasian females, which may limit generalizability to other ethnic or racial groups. Nonetheless, this study represents the largest cohort of AYA females with CHD with menstrual related data.

The large number of complaints about menstrual dysfunction but little use of hormonal medication, inappropriate use of estrogen, and potential overuse of OTC medication indicates menstrual dysfunction is inadequately addressed in this population. The lack of reported hormonal contraception use among those who have previously been sexually active indicates contraceptive needs are not being adequately addressed. As medical treatments continue to expand longevity of women with CHD, we predict the need to address these reproductive issues will also increase. ${ }^{13} \mathrm{~A}$ precision health approach would benefit this population with their unique and complex needs. Next steps would include further education for primary care providers and cardiologist to assist in identifying reproductive needs for direct intervention or referral to providers that feel comfortable with their gynecological needs of AYA females with 
CHD. Cardiologists may consider collaborating with reproductive health specialists such as gynecologists and adolescent health providers to best provide for these patients.

\section{Disclosure/Conflict of Interest Statement:}

Authors have nothing to disclose. 


\section{References:}

1. Hargrove A, Penny DJ, Sawyer SM. Sexual and Reproductive Health in Young People with Congenital Heart Disease: A Systematic Review of the Literature. Pediatric Cardiology. 2005;26(6):805-811.

2. Mendelson MA. Gynecologic and obstetric issues in the adolescent with heart disease. Adolescent medicine (Philadelphia, Pa). 2001;12(1):163-174.

3. Somerville J. Congenital heart disease in the adolescent. Arch Dis Child. 1989;64(6):771-773.

4. Thorne S, MacGregor A, Nelson-Piercy C. Risks of contraception and pregnancy in heart disease. Heart. 2006;92(10):1520-1525.

5. Vigl M, Kaemmerer M, Seifert-Klauss V, et al. Contraception in women with congenital heart disease. Am J Cardiol. 2010;106(9):1317-1321.

6. Canobbio MM, Perloff JK, Rapkin AJ. Gynecological health of females with congenital heart disease. Int J Cardiol. 2005;98(3):379-387.

7. Roe AH, Dutton C. Contraception for Transplant Patients. Transplantation. 2017;101(8):17391741.

8. Osteen KA, Beal CC. Reproductive Health and Women With Congenital Heart Disease: A Practice Update. The Journal of perinatal \& neonatal nursing. 2016;30(1):25-35.

9. Reid GJ, Siu SC, McCrindle BW, Irvine MJ, Webb GD. Sexual behavior and reproductive concerns among adolescents and young adults with congenital heart disease. Int J Cardiol. 2008;125(3):332-338.

10. Kaemmerer M, Vigl M, Seifert-Klauss V, et al. Counseling reproductive health issues in women with congenital heart disease. Clinical research in cardiology : official journal of the German Cardiac Society. 2012;101(11):901-907.

11. Uzark K, VonBargen-Mazza P, Messiter E. Health education needs of adolescents with congenital heart disease. Journal of pediatric health care : official publication of National Association of Pediatric Nurse Associates \& Practitioners. 1989;3(3):137-143.

12. Dore A, de Guise P, Mercier LA. Transition of care to adult congenital heart centres: what do patients know about their heart condition? The Canadian journal of cardiology. 2002;18(2):141146.

13. Meschke LL, Bartholomae S, Zentall SR. Adolescent sexuality and parent-adolescent processes: promoting healthy teen choices. J Adolesc Health. 2002;31(6 Suppl):264-279.

14. Miller KS, Kotchick BA, Dorsey S, Forehand R, Ham AY. Family communication about sex: what are parents saying and are their adolescents listening? Fam Plann Perspect. 1998;30(5):218-222, 235.

15. Ramchandani K, Morrison P, Gold MA, Akers AY. Messages About Abstinence, Delaying Sexual Debut and Sexual Decision-Making in Conversations Between Mothers and Young Adolescents. Journal of pediatric and adolescent gynecology. 2018;31(2):107-115.

16. Curtis KM, Tepper NK, Jatlaoui TC, et al. U.S. Medical Eligibility Criteria for Contraceptive Use, 2016. MMWR Recommendations and reports : Morbidity and mortality weekly report Recommendations and reports. 2016;65(3):1-103.

17. Stout KK, Daniels CJ, Aboulhosn JA, et al. 2018 AHA/ACC Guideline for the Management of Adults With Congenital Heart Disease: A Report of the American College of Cardiology/American Heart Association Task Force on Clinical Practice Guidelines. Circulation. 2019;139(14):e698e800.

18. Division of Reproductive H, Centers for Disease C, Prevention, et al. U S. Medical Eligibility Criteria for Contraceptive Use, 2010: adapted from the World Health Organization Medical Eligibility Criteria for Contraceptive Use, 4th edition. MMWR Recommendations and reports : 
Morbidity and mortality weekly report Recommendations and reports / Centers for Disease Control. 2010;59(RR-4):1-86.

19. Silversides CK, Sermer M, Siu SC. Choosing the best contraceptive method for the adult with congenital heart disease. Current cardiology reports. 2009;11(4):298-305.

20. Warnes CA, Williams RG, Bashore TM, et al. ACC/AHA 2008 Guidelines for the Management of Adults with Congenital Heart Disease: a report of the American College of Cardiology/American Heart Association Task Force on Practice Guidelines (writing committee to develop guidelines on the management of adults with congenital heart disease). Circulation. 2008;118(23):e714-833.

21. Stout KK, Daniels CJ, Aboulhosn JA, et al. 2018 AHA/ACC Guideline for the Management of Adults With Congenital Heart Disease: A Report of the American College of Cardiology/American Heart Association Task Force on Clinical Practice Guidelines. J Am Coll Cardiol. 2019;73(12):e81e192.

22. Neinstein LS, Katzman D, Callahan T. Neinstein's adolescent and young adult health care : a practical guide. 6th edition. ed. Philadelphia, PA: Wolters Kluwer; 2016.

23. World Health Organization Task Force on Adolescent Reproductive H. World Health Organization multicenter study on menstrual and ovulatory patterns in adolescent girls: II. Longitudinal study of menstrual patterns in the early postmenarcheal period, duration of bleeding episodes and menstrual cycles. Journal of Adolescent Health Care. 1986;7(4):236-244.

24. Flug D, Largo RH, Prader A. Menstrual patterns in adolescent Swiss girls: a longitudinal study. Annals of Human Biology. 1984;11(6):495-508.

25. Day RO, Snowden L, McLachlan AJ. Life-threatening drug interactions: what the physician needs to know. Internal Medicine Journal. 2017;47(5):501-512.

26. Peake LJ, Grover SR, Monagle PT, Kennedy AD. Effect of warfarin on menstruation and menstrual management of the adolescent on warfarin. J Paediatr Child Health. 2011;47(12):893897.

27. Van Eijkeren MA, Christiaens GC, Sixma JJ, Haspels AA. Menorrhagia: a review. Obstet Gynecol Surv. 1989;44(6):421-429.

28. Ferreira M, Barsam S, Patel JP, et al. Heavy menstrual bleeding on rivaroxaban. British Journal of Haematology. 2016;173(2):314-315.

29. Beyer-Westendorf J, Michalski F, Tittl L, Hauswald-Dörschel S, Marten S. Vaginal bleeding and heavy menstrual bleeding during direct oral anti-Xa inhibitor therapy. Thrombosis and haemostasis. 2016;115(06):1234-1236.

30. Lauring JR, Lehman EB, Deimling TA, Legro RS, Chuang CH. Combined hormonal contraception use in reproductive-age women with contraindications to estrogen use. American Journal of Obstetrics \& Gynecology. 2016;215(3):330.e331-330.e337.

31. Yu J. HX. Inappropriate Use of Combined Hormonal Contraceptives for Birth Control Among Women of Reproductive Age in the United States. Journal of Women's Health. 2013;22(7):595603. 
Table 1. Survey questions and responses.

\begin{tabular}{|c|c|}
\hline Reproductive Health Questions (N=114) & $\begin{array}{l}\text { Yes } \\
\mathbf{N}(\%)\end{array}$ \\
\hline \multicolumn{2}{|c|}{ Have you had any problems with the following menstrual (period) related issues: } \\
\hline Cramping? & $77(68)$ \\
\hline Irregular periods? & $48(42)$ \\
\hline Heavy periods & $53(47)$ \\
\hline $\begin{array}{l}\text { Have you (or your daughter) seen a clinician (doctor or nurse) for a } \\
\text { menstrual problem? }\end{array}$ & $33(29)$ \\
\hline $\begin{array}{l}\text { Have you (or your daughter) taken a medicine for pain relief that you } \\
\text { can get over the counter such as acetaminophen, ibuprofen, naproxen } \\
\text { or brand name medications (such as Tylenol }(\text {, Midol }(\AA \text {, Advil }(\text {, } \\
\text { Aleve } \Theta) \text { ? }\end{array}$ & $95(83)$ \\
\hline $\begin{array}{l}\text { Have you (or your daughter) taken any hormones (birth control pills, } \\
\text { estrogen, progestin, etc.) for a menstrual problem or for puberty? }\end{array}$ & $38(33)$ \\
\hline Have you ever had sex with a male (penis in the vagina)? $?^{*}$ & $34(34)$ \\
\hline 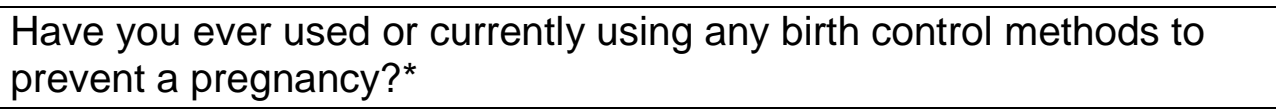 & $19(19)$ \\
\hline \multicolumn{2}{|c|}{ Have you (Has your daughter) ever seen a health provider for any of the below reasons? } \\
\hline Menstrual suppression (stop my periods) for cramps & $11(9.6)$ \\
\hline Menstrual suppression (stop my periods) for heavy bleeding & $15(13.2)$ \\
\hline Birth control to help prevent a pregnancy & $16(14.0)$ \\
\hline I (She) was pregnant & $3(2.6)$ \\
\hline I have not seen a health provider for these conditions & $80(70.2)$ \\
\hline
\end{tabular}

${ }^{\star} \mathrm{N}=99$, question not asked of parents. 
Table 2. Participants demographics and $\mathrm{CHD}$ lesions $(\mathrm{N}=114)$.

\begin{tabular}{|l|c|}
\hline & Mean (SD) or N (\%) \\
\hline Age (years, range 14-21) & $17.0 \pm 2.2$ \\
\hline Race (White) & $99(88 \%)$ \\
\hline Age at menarche & $12.5 \pm 1.7$ \\
\hline Ever had sex (yes) $^{\star}$ & $34(34 \%)$ \\
\hline CHD Complexity ${ }^{\star *}$ & $28(24.6 \%)$ \\
\hline Simple & $46(40.4 \%)$ \\
\hline Moderate & $33(28.9 \%)$ \\
\hline Complex & $7(6.1 \%)$ \\
\hline Confirmed transplant & \\
\hline
\end{tabular}

${ }^{*} \mathrm{~N}=99$, question not asked of parents

${ }^{* *}$ Complexity determined by American College of Cardiology 


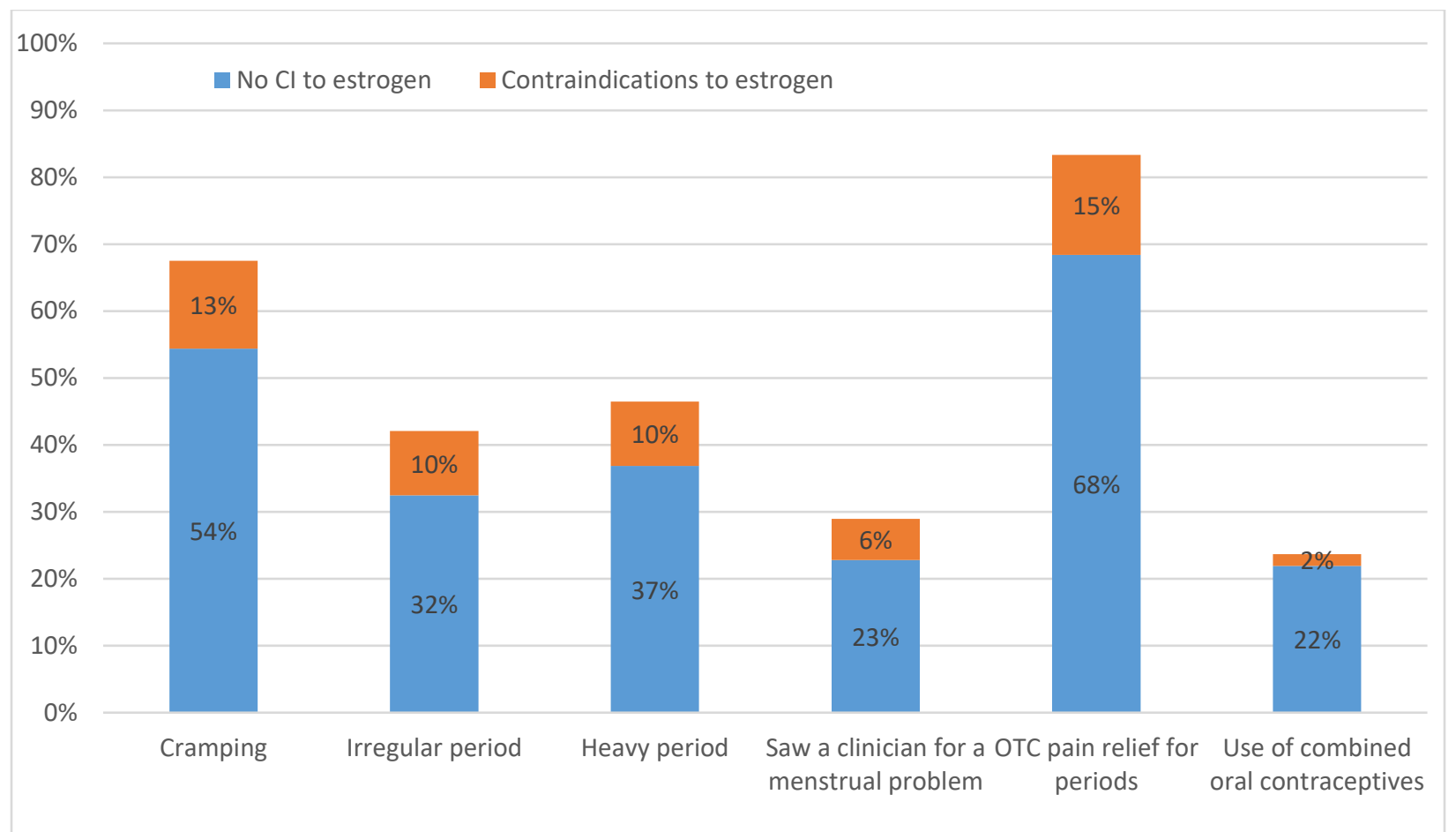

Figure 2. Self-reported menstrual concerns and treatments $(\mathrm{N}=114)$. 


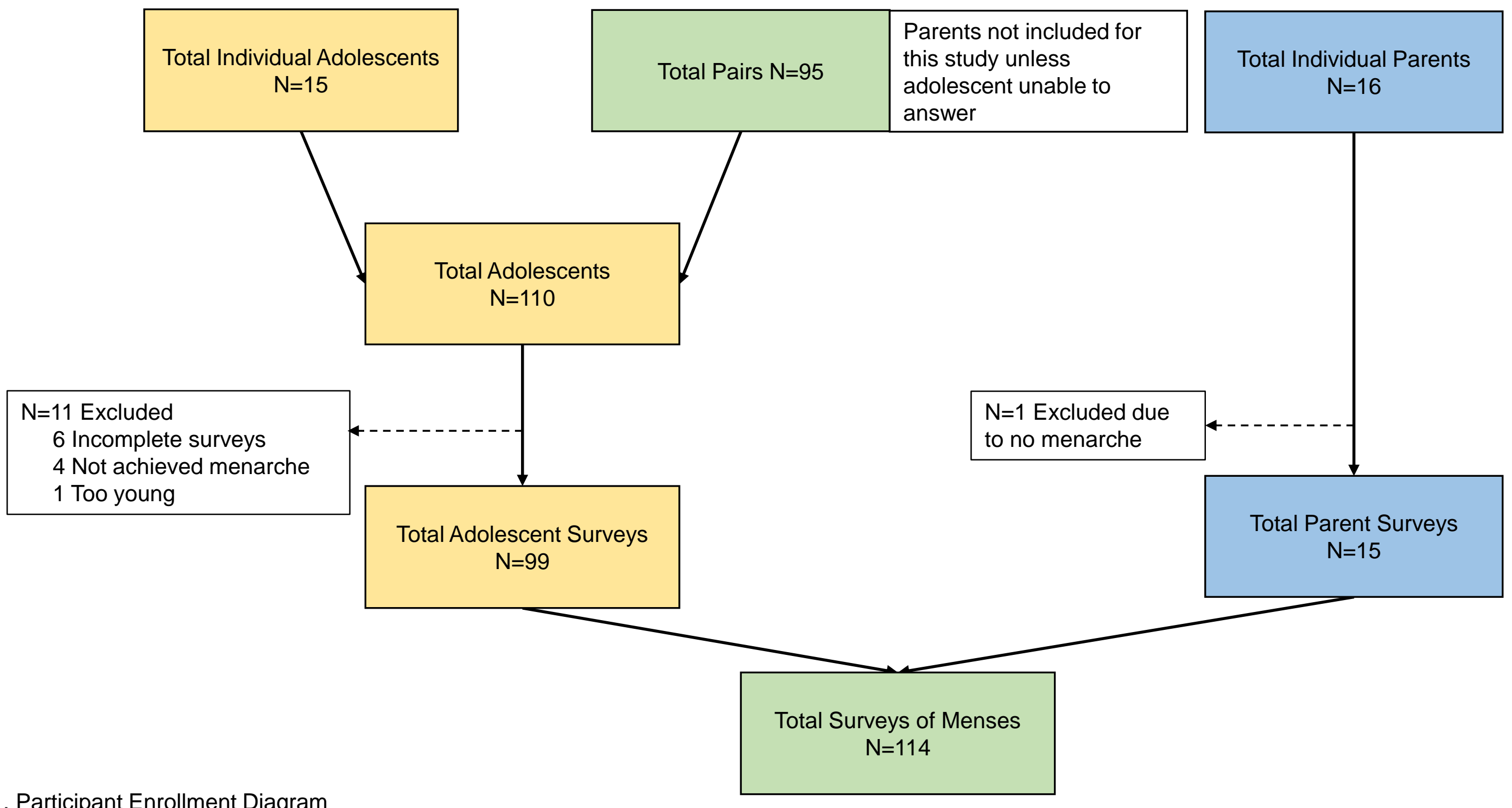

